\title{
Research of organizational and technological features of construction using modeling
}

\author{
Marat Kuzhin* \\ Moscow State University of Civil Engineering, Yaroslavskoe shosse, 26, Moscow, 129337, Russia
}

\begin{abstract}
The article deals with the organizational and technological features of construction. Methods of optimization of values to the required level are considered. Recommendations are given on making changes to organizational and technological solutions and achieving the required indicators. The article presents an attempt to study the issue related to the modeling of construction in general and its individual stages, including construction production. The main elements related to planning and construction management are considered. Various approaches, methods and methods of studying the issues of modeling in relation to the building system are presented. Due to the fact that construction is a complex system with a large number of subjects, various interrelationships between them, constantly changing in the process and poorly regulated by regulatory and technical documents, modeling a construction system is a difficult task. The task of adjustment is reduced to the compilation of an optimal work schedule, ensuring the greatest uniformity of resource schedules. The correct adjustment of the schedules is based on the calculation of the coefficients and parametric indicators of the resource schedules. Comparing them with normal indicators, we can conclude that the optimization was successful. New technologies make it possible to take into account a large number of non-standard solutions that allow the development of various options and which can be applied depending on the type of initial data and the conditions for organizing construction production. The process of planning and analyzing construction information using aggregation can be useful if it allows you to get rid of redundant information that is not particularly important. Also, the use of this method is justified when analyzing large amount of information. An example of the use of aggregation is the transition to the sum, to the average, if you want to aggregate indicators. Aggregation also applies to graphical models (such as flowcharts) and in many other cases.
\end{abstract}

\section{Introduction}

Construction is a branch of the national economy, in the management of which a huge number of participants are involved, including from related industries. Construction takes place in a constantly changing environment. Therefore, planning must take into account the

\footnotetext{
* Corresponding author: kuzhinmf@mail.ru
} 
dynamic component of individual controls and lay a certain degree of flexibility. Specialists from the economic, financial, transport and construction sectors are involved in organizing the construction of buildings and structures. In order for this complex system where the elements of the process are interconnected and mutually influence each other, to function according to the developed plan [1,2]. The calculation of the parameters of construction project is carried out on the basis of the conditions that each work is provided with all the necessary resources. In order to effectively manage the construction process, it is necessary to have a complete visual picture of what is happening at each specific stage of construction. One of the most important elements of construction management is the simulation of the construction process - modeling. Modeling is a rather difficult formalized area of a manager's activity, which is considered the art of management. The manager's awareness of the fact that he is modeling allows him to: systematize actions; analyze the reasons for failure and success; accumulate and transfer their experience and knowledge; justify and explain their decisions. Thus, modeling allows the manager to conduct an effective analysis of the situation and make informed decisions.

\section{Materials and methods}

The research of the issue was carried out on the basis of the analysis of theoretical data adopted in the organizational and technological documentation. During the study, a comparison of optimization methods was performed. Criteria assessment or application of a generalized indicator depends on the goals of the project and the tasks to be solved.

\section{Results}

This research is devoted to the planning, organization and management of construction, the stage of an investment project associated with construction and not design and preinvestment stage. The main problem of construction planning is that many decisions in construction are made according to the situation in a working order. Only certain stages of construction and the general terms of work are planned in advance. This raises a large number of questions of a different nature. Some of the decisions you make can be difficult, others can be simple. But the main planning complexity is not caused by the decisions themselves, but rather by their interconnectedness. Due to the fact that construction is a complex system with a large number of subjects, various interrelationships between them, constantly changing in the process and poorly regulated by regulatory and technical documents, a systematic approach to the analysis of the management and planning structure should be applied. In addition, when analyzing the structure of management of construction processes, aggregation and disaggregation can be applied. The process of planning and analyzing construction information using aggregation can be useful if it allows to get rid of redundant information that is not particularly important. Also, the use of this method is justified when analyzing large amounts of information. An example of the use of aggregation is the transition to the sum, to the average, if you want to aggregate indicators. Aggregation also applies to graphical models (such as flowcharts) and in many other cases.

In practice, there many directions of systems modeling but one of the most closely describing a building system is the dynamic model, which include multilevel functional dependencies, imitation of the organizational process and formalization of interrelationships between individual elements, modeling the organizational behavior of real building systems and statistical analysis of data from construction sites, collection and processing of information. A dynamic model includes variables that change over time. Typically, these models contain various differential equations. A model can be viewed as dynamic if at least one of the variables depends on time. In the general case, the dynamic model describes the state of the building system, technological, organizational and financial 
relationships, while taking into account the possible optimization according to the specified criterion. The mathematical description of dynamic models is based on the use of differential equations. In the process of studying dynamic models, the influence of external factors and the stability of the model on the constructed model is of great practical interest. The main goal is to maintain the overall structure of the system, adhere to the performance criteria and achieve the project results with the planned indicators. At the same time, the tasks of solving optimization problems, which are usually used in solving local problems of the project, remain important.

Modeling processes at certain levels is a complex task that should be performed through scientific research of the features and identification of the interrelationships of processes, determination of influencing factors and their formalization $[3,4,5,6]$. The distinction between the levels is associated with the nature of the tasks being solved, in which the processes may not have a direct connection. Modeling is an important component of construction management or its individual stages and allows you to increase the level of consistency of decisions of the participants in the construction industry and make more accurate decisions. Despite the fact that the models of the organization of production are not mandatory for adherence, nevertheless the systematization and structuring of processes and bringing them repetitive controlled and predictable actions is necessary to improve efficiency and effectiveness [7,8,9,10,11]. A particular difficulty in modeling is the diversity of the elements of the construction process, the non-periodicity and formalization of individual elements, without which the beginning of another process is impossible. Individual elements, such as the actions of some participants in the construction, also present the complexity of formalization. For example, the suspension of construction in connection with imposed penalties related to violations of safety requirements, environmental requirements, fire safety requirements or other restrictions of a similar nature. The heterogeneity of the studied and formulated relationships imposes additional constraints on modeling.

In order to achieve stable dynamics of construction, it is necessary to take into account the uniform distribution of resources when designing. Resource schedules are designed to solve this problem. To consider the main elements of modeling organization, planning and construction management $[11,12,13]$. Construction in general can be divided into several major levels:

1) management of construction projects;

2) organization of construction production;

3) technological features of construction work.

In order to develop a management model for each of the stages of construction, it is necessary to determine the main elements of this system and the tasks to be solved at these stages, indicating the participants, terms and other parameters of construction (Fig. 1). 


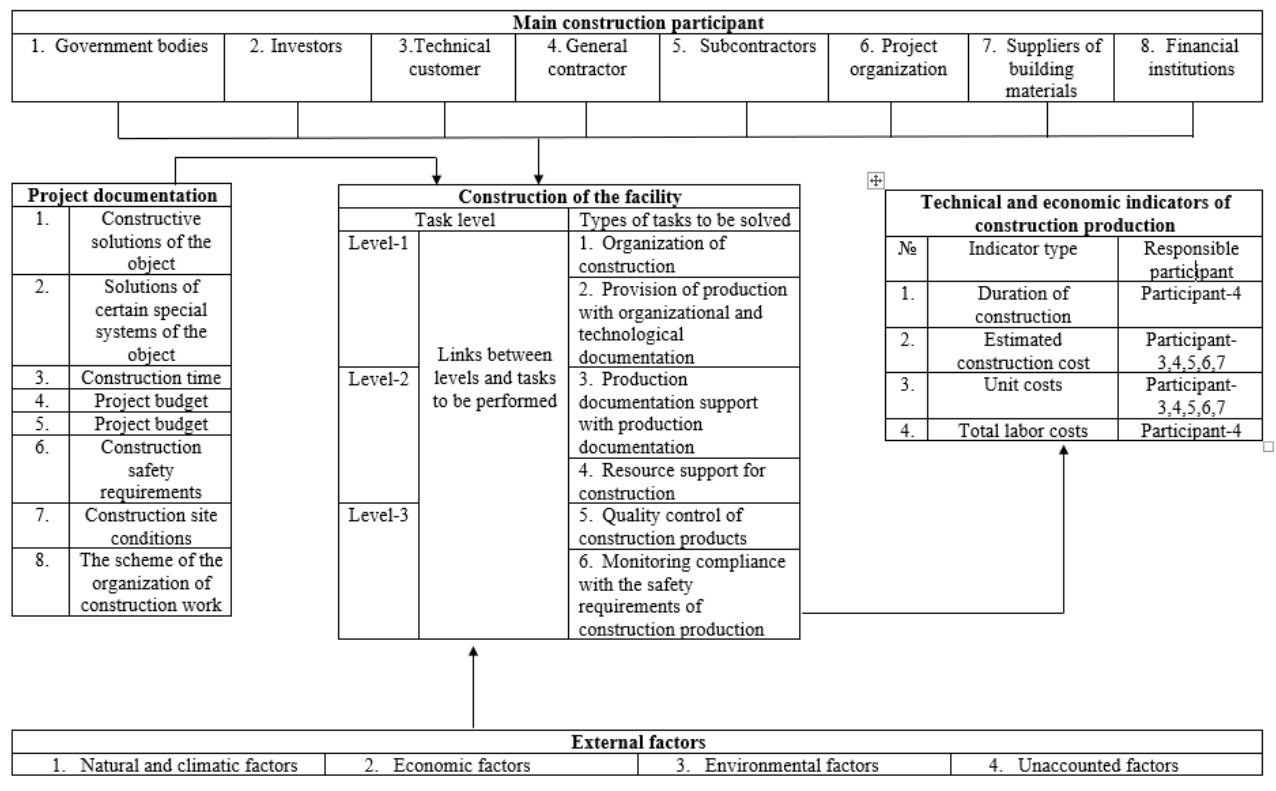

Fig. 1. Simplified diagram of the organization of construction production

The above scheme presents a general scheme of interaction of the main participants in construction, management levels and the main technical and economic parameters and indicators of construction processes. The diagram shows the main elements that have a significant impact on the organization of the construction process. Hierarchical structures are formed to streamline connections between system elements. Management in a system with a hierarchical structure is based on the fact that each of the subsystems solves some particular problem under conditions of relative independence. To model the structure of an organization, two types of model are used: analytical; graphic. The research is aimed at studying the management of construction processes and studying the nature of their influence and interaction on the performance of work and construction in general. The study of numerical values and the construction of functions of construction processes as a whole as a system is not included in the scope of this study and will be continued at the next stage. In the study, an attempt was made to study the individual elements of the building system, to build a hierarchy of mutual relations between the participants, the object under construction and the parameters of the work.

All of the above tasks (Fig. 1) of the construction industry can be formalized and a matrix of parameters can be constructed (Table 1). 
Table 1. Construction production parameters matrix

\begin{tabular}{cccccc}
\hline $\begin{array}{c}\text { Group of } \\
\text { tasks to be } \\
\text { solved }\end{array}$ & Level -1 & Level -2 & Level -1 & $\begin{array}{c}\text { The current level } \\
\text { of implementation, } \\
\%\end{array}$ & Required level \\
\hline 1 & A1 & B1 & C1 & K1 & D1 \\
2 & A2 & B2 & C2 & K2 & D2 \\
3 & A3 & B3 & C3 & K3 & D3 \\
4 & A4 & B4 & C4 & K4 & D4 \\
5 & A5 & B5 & C5 & K5 & D5 \\
6 & A6 & B6 & C6 & K6 & D6 \\
$\begin{array}{c}\text { Total for } \\
\text { the project }\end{array}$ & F1 & F2 & F3 & - & -
\end{tabular}

The parameters that are formalized in the table for examples can be divided into 2 large groups: controlled and random. In addition, parameters and indicators can be qualitative or quantitative. To process an array of current data, you can apply mathematical methods and software products in order to automate calculations. Existing software products for planning automation are used in the development of information models for construction. Some of the programs contain a system of statistical data analysis, which includes a wide range of analytical procedures and methods, including: construction of various types of graphs; collection and analysis of static information; multiple regression calculations; nonparametric statistics; exploratory data analysis, correlations; interactive probabilistic calculator; tables of frequencies, contingencies, flags and headers; fitting distributions and much more. At the same time, software systems allow: collecting and storing data; working with variables of different types; recognize the frequency of signs, tables, graphs, contingency tables, diagrams; denote primary descriptive statistics; conduct marketing research and analysis of the data obtained from these studies. The computer system aided design systems, focused on the preparation of interactive documents with calculations and visual support, is intended for symbolic calculations, although it has a number of tools for the numerical solution of differential equations and finding integrals $[15,16]$.

The groups of parameters (types of tasks to be solved) presented in table 1 can be classified according to their functional characteristics.

1) organization of construction production (solving organizational issues related to interaction with construction participants in general; organization of the construction of the facility as a whole; organization of certain types of construction work);

2) support of production with organizational and technological documentation (development of a construction organization project; development of a project for the production of works; development of technological maps);

3) documentation support of production with production documentation (General organizational documents; production documentation; production documentation);

4) resource support for construction (control of contractual relations; resource provision of construction production; control of resource consumption by type of work);

5) quality control of construction products (quality control of a complex of objects; check in the aggregate of individual stages of construction and the entire object; check the quality of certain types of construction work);

6) monitoring compliance with the safety requirements of construction production (development of recommendations for a construction organization during the construction of facilities; compliance with safety requirements for the construction site as a whole and 
areas indicated on the construction master plan ; organization of a workplace and work with tools when performing certain types of construction work).

Modeling of construction production can be performed using symbolic elements or mathematical ones. Symbolic models most clearly reflect the basic elements of all processes. The main types of symbolic models are work production schedules, which can be presented in several basic forms:

linear work schedule; network schedule of work performance; cyclogram (line graph view).

Each of the presented forms has its own advantages and disadvantages, and therefore has a certain field of application. The linear form is the simplest and clearly shows the progress of construction as a whole. In this model, the parameters of each individual work (list of work, scope of work, labor intensity, numerical-qualification composition of workers, duration of work) and the graphic part in the form of horizontal lines showing the progress of work are presented. The linear form is used in the development of almost all basic organizational and technological documents, including a technological map, work production projects and construction organization projects. The network form is used less often than the linear form, but it has its own purpose. As a rule, this form is used for tasks related to construction management. This is especially important for the organization of interaction between the participants in the construction and the designation of the relationship between the works. The cyclogram as a type of linear form serves to solve local problems at the construction site. It is effective, if necessary, to organize work and the distribution of teams of workers on the fronts of work for their continuous execution. Mathematical modeling is advisable to apply when there is a need to accurately calculate all the parameters and predict the probability of their occurrence. Mathematical modeling allows you to take into account the relationship between groups of parameters and assess the impact on each other, to visualize these relationships on the graphs of dependence. However, a big disadvantage of mathematical modeling is that it is difficult to visualize all the elements of the organization of construction production with all its participants, since some interactions are difficult to represent in the form of mathematical dependencies. One of the main directions in the development of the construction industry is the development of a model that clearly shows the progress of construction, the relationship between its elements and takes into account the parametric data of processes and system elements. In some cases, it is necessary to approach the solution of organizational tasks separately, in particular to each one and already take into account the elements and parameters of the model that are important for this stage of decision-making.

During processing complex descriptions with significant amounts of information, schemes based on sequential aggregation and disaggregation are often used. In practice, aggregation takes the form of transforming the original model into an aggregated model, i.e. with fewer variables or constraints. The aggregated model gives an approximate description of an economic object or process compared to the initial one. Systems analysis is important in modeling. System analysis can be represented as a set of techniques for solving problems arising in purposeful activity, based on the use of systemic methodology. System analysis is characterized in two ways: it is a scientific discipline that develops general principles for the study of complex objects, taking into account their systemic nature; that is the methodology of studying objects by means of their representation as systems and analysis of these systems. Systems analysis is mainly associated with the study of human organizations, while it involves not only analysis, but also design, that is, the development, restructuring of management systems. At the same time, being a method for studying objects, system analysis is an effective means of solving complex, insufficiently clearly formulated problems, including in the economy. Each object is considered as a system consisting of components. 
Any model aims to establish an unambiguous understanding or assessment of the situation in question for making a decision. Decision-making errors occur for several reasons: information about the situation; assessment of the information received; assessment of the situation based on perceived information; solution method; assessment of the consequences of decisions made.

The consequences of mistakes in decision making in the economic are so large that in order to avoid them, economic and mathematical modeling is used. Economic and mathematical modeling is a manager's tool and aims to make informed decisions and assess their consequences. Large systems are complex and intertwined with organizational and informational links $[17,18]$. Hierarchical structures are formed to streamline connections between system elements. Hierarchy is a type of structure, organization of a system, based on the ordering of its elements from the highest to the lowest and the introduction of subordination relations. Hierarchical structures are widely used in control systems and classifications $[19,20]$. Management in a system with a hierarchical structure is based on the fact that each of the subsystems solves some particular problem under conditions of relative independence. It is impossible to talk about the existence of a strict hierarchy in reality, since in economic and social systems there are connections between elements that are not provided for by such a structure model as a tree. Exploratory analysis of multidimensional data is used to build classifications and build hierarchies. It is used if the researcher has a table of multidimensional data characterizing the structure, and, at the same time, there is no information about the causal mechanism for generating this data. The structure of construction production management (linear, functional or matrix) affects the modeling scheme of the entire construction process.

\section{Conclusion}

Modeling construction production is a large task based on the use of mathematical apparatus and parametric equations. The developed model should take into account the dynamic parameters of the system and the variability of event outcomes. In this case, an important role is played by the process of optimizing solutions according to various criteria. Thus, the task of adjustment is reduced to the compilation of an optimal work schedule, ensuring the greatest uniformity of resource schedules. The correct adjustment of the schedules is based on the calculation of the coefficients and parametric indicators of the resource schedules. Comparing them with normal indicators, we can conclude that the optimization was successful. New technologies make it possible to take into account a large number of non-standard solutions that allow the development of various options and which can be applied depending on the type of initial data and the conditions for organizing construction production. The automation of the construction modeling process is already in progress and is coming into play, but there are still many areas that require manual control. Improving the modeling of such sites will allow avoiding unforeseen situations and stopping the construction process. The other side of the need for modeling is to improve the understanding of the main stages of construction by the management participants and increase the efficiency of interaction.

\section{References}

1. Lapidus A., Abramov I. Implementing large-scale construction projects through application of the systematic and integrated method XXIst International Scientific Conference on Advanced in Civil Engineering: Construction - The Formation of Living Environment, FORM 2018. "IOP Conference Series: Materials Science and Engineering" 2018. P. 062002. 
2. Lozhkomoeva E.N. Improvement of the organizational and economic mechanism of management of a machine-building enterprise and its adaptation to changes in the market environment. dissertation abstract for the degree of candidate of economic sciences / Moscow State University of Instrument Engineering and Informatics. Moscow, 2012.

3. M.Kuzhin, B.Zhadanovsky The choice of effective organizational and technological solutions for the installation of hinged facade systems during the overhaul of residential buildings. Technology and organization of construction production. 2012. No. 1-1. S. 47-49.

4. B.Zhadanovsky S.Sinenko, M.Kuzhin Analysis of the data required for the organizational and technological design of the reconstruction of buildings and structures Technology and organization of construction production. 2014. No. 3. S. 43-45.

5. B.Zhadanovsky, S.Sinenko, M.Kuzhin Rational organizational and technological schemes for the production of construction and installation works in the conditions of reconstruction of an operating enterprise Technology and organization of construction production. 2014. No. 1. S. 38-40.

6. Topchy D.V., Lapidus A.A. Construction supervision at the facilities renovation Topical Problems of Architecture, Civil Engineering and Environmental Economics (TPACEE 2018) electronic edition. Ser. "E3S Web of Conferences" 2019. P. 08044.

7. Lapidus A., Shesterikova Y. Mathematical model for assessing the high-rise apartment buildings complex quality E3S Web of Conferences 2019. P. 02025.

8. Sinenko S.A., Ginzburg V.M., Sapozhnikov V.N., Kagan P.B., Ginzburg A.V. Automation of organ. and technolog. design in construction: Textbook.- Saratov: Higher education, 2019. -235p.

9. Lapidus A., Abramov I. A system-integrated approach to the study of the problem of ensuring the sustainability of complex production and dynamic systems in construction / In the collection: Construction system engineering. Cyberphysical building systems A collection of materials from a seminar held as part of the VI International Scientific Conference. 2018.S. 159-162.

10. Abramov I, Lapidus A. Systemic integrated method for assessing factors affecting construction timelines Collected at: International Scientific Conference Environmental Science for Construction Industry - ESCI 2018 Ser. "MATEC Web of Conferences" 2018.S. 05033.

11. Oleinik P.P. The choice of the rational relationship of the method and form of organization of construction Industrial and civil construction. 2019. No 6.P. 46-50.

12. Pakhomova L.A., Oleinik P.P. Selection and assessment of parameters for certification of work places sout (special assessment of working conditions) Technology and organization of construction production. 2019. No 1. S. 49-52.

13. Oleinik P., Yurgaytis A., Voronina G., Makarenko A. Methods for the formation and optimization of calendar plans for construction companies Collected: MATEC Web of Conferences 2018.S. 05037.

14. Zhadanovsky B.V., Erzhokova E.S., Gorshkova E.A. Stream method as a way of organizing the construction of System Technologies. 2018. No. 3 (28). S. 136-140.

15. Zelentsov A.A., Tokarsky A.Ya. Modeling the consequences of the risks of manifestation of negative factors during the construction of capital construction projects. In the collection: Days of student science. Collection of reports of a scientific and technical conference based on the results of research work by students of the 
Institute of Construction and Architecture. 2019.S. 1361-1363.

16. Lapidus A.A., Tolstova K.S., Topchiy D.V. Formation of groups of parameters affecting the criterion of admissibility of combining processes in the production of finishing works Science and business: development paths. 2018.No 6 (84). S. 18-22.

17. Solomatina M.I. Study of the influence of destabilizing factors on the reliability of production processes. In the collection: Days of student science. Collection of reports of a scientific and technical conference based on the results of research work by students of the Institute of Construction and Architecture. 2019.S. 1299-1301.

18. Liu, J., \& Lu, M. (2020). Synchronized optimization of various management-function schedules in a multiproject environment: Case study of planning steel girder fabrication projects in bridge construction. Journal of Construction Engineering and Management, 146(5) doi:10.1061/(ASCE)CO.1943-7862.0001813.

19. Arashpour, M., Kamat, V., Bai, Y., Wakefield, R., \& Abbasi, B. (2018). Optimization modeling of multi-skilled resources in prefabrication: Theorizing cost analysis of process integration in off-site construction. Automation in Construction, 95, 1-9. doi:10.1016/j.autcon.2018.07.027.

20. Sun, Q., Li, D., \& Ren, Y. (2018). Study of redundancy and variance based P-cycle construction algorithm. Tiedao Xuebao/Journal of the China Railway Society, 40(12), 101-107. doi:10.3969/j.issn.1001-8360.2018.12.013 